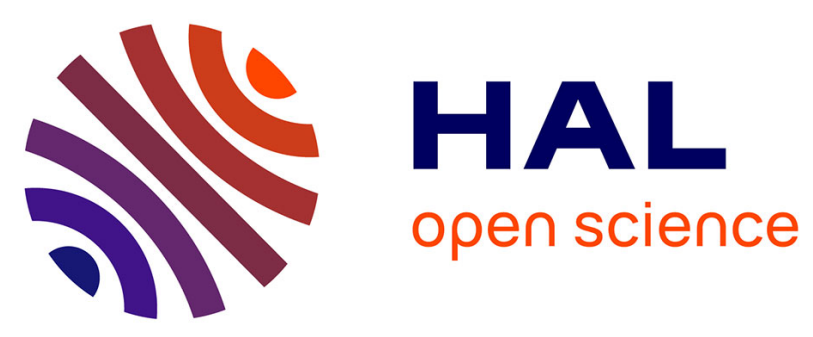

\title{
Pouvoir princier et structures de la propriété immobilière à Milan au temps des Sforza (1450-1500) : questions et perspectives
}

Patrick Boucheron

\section{- To cite this version:}

Patrick Boucheron. Pouvoir princier et structures de la propriété immobilière à Milan au temps des Sforza (1450-1500) : questions et perspectives. Faron, Olivier; Hubert, Etienne. Le sol et l'immeuble: les formes dissociées de propriété immobilière dans les villes de France et d'Italie: XIIe-XIXe siècle: actes de la table ronde organisée par le Centre interuniversitaire d'histoire et d'archéologie médiévales et le Centre Pierre Léon (Université Lumière Lyon 2, Ecole des hautes études en sciences sociales, CNRS) avec le concours de l'Ecole française de Rome: Lyon, 14-15-mai 1993, Centre interuniversitaire d'histoire et d'archéologie médiévales: Presses universitaires de Lyon / Ecole française de Rome, Palais Farnèse, pp.207-226, 1995. hal-03264933

\section{HAL Id: hal-03264933 https://hal.science/hal-03264933}

Submitted on 18 Jun 2021

HAL is a multi-disciplinary open access archive for the deposit and dissemination of scientific research documents, whether they are published or not. The documents may come from teaching and research institutions in France or abroad, or from public or private research centers.
L'archive ouverte pluridisciplinaire HAL, est destinée au dépôt et à la diffusion de documents scientifiques de niveau recherche, publiés ou non, émanant des établissements d'enseignement et de recherche français ou étrangers, des laboratoires publics ou privés. 


\section{Pouvoir princier et structures de la propriété immobilière à Milan au temps des Sforza (1450-1500) : questions et perspectives} Patrick Boucheron

\section{Résumé}

La documentation publique jette sur les structures de la propriété urbaine dans la capitale lombarde au XVe siècle une lumière contrastée qui, si elle n'éclaire pas l'ensemble des phénomènes du marché immobilier, en détaille les évolutions que le pouvoir princier tente de contrôler. On y observe d'abord les limites frangées des espaces publics et des espaces privés, dont l'imbrication et l'enchevêtrement nuancent la représentation qu'en donnent les sources statutaires. C'est d'ailleurs en jouant de ces chevauchements que le pouvoir princier tente, au-delà de son rôle traditionnel de défense de l'espace public, de mettre un pied dans l'édification privée. Mais la complexification des régimes de propriété représente également un défi pour l'effort réglementaire D'où la multiplication, de 1456 à 1466, des lettres de concessions ducales que l'on étudie ici, et qui révèlent l'étendue des formes dissociées de la propriété à Milan en même temps que la difficulté que pose leur contrôle politique.

\section{Citer ce document / Cite this document :}

Boucheron Patrick. Pouvoir princier et structures de la propriété immobilière à Milan au temps des Sforza (1450-1500) : questions et perspectives. In: Le sol et l'immeuble. Les formes dissociées de propriété immobilière dans les villes de France et d'Italie (XIle-XIXe siècle) Rome : École Française de Rome, 1995. pp. 207-226. (Publications de l'École française de Rome, 206);

https://www.persee.fr/doc/efr_0223-5099_1995_act_206_1_5943

Fichier pdf généré le 13/09/2018 


\section{POUVOIR PRINCIER ET STRUCTURES DE LA PROPRIÉTÉ IMMOBILIËRE À MILAN AU TEMPS DES SFORZA (1450-1500) : QUESTIONS ET PERSPECTIVES}

L'emprise politique du pouvoir communal sur le sol urbain constitue une des questions les plus classiques de l'histoire des villes italiennes. La définition d'un espace public comme domaine propre de l'autorité commune, dégagé de la logique d'appropriation foncière et protégé des empiétements du privé, est apparue depuis longtemps comme une expression majeure de la réalité et du fonctionnement de ce type de pouvoir. De ce point de vue, le passage à la seigneurie ne modifie pas radicalement les données du problème : investissant les rouages de l'administration communale, les princes d'Italie du Nord reprennent à leur compte leurs aspirations politiques et leurs pratiques administratives. Mais l'État princier ne peut toutefois se cantonner dans une politique défensive, laissant hors de portée réglementaire les mécanismes d'un marché immobilier en voie de constitution. Dans leur capitale, les ducs de Milan cherchèrent avec obstination les moyens politiques de contrôler l'ensemble du sol urbain. Ce faisant, ils devaient se glisser dans l'entrelacs subtil des formes dissociées de la propriété où l'espace public et l'espace privé s'enchevêtrent en un tissu complexe. Cette ambition politique, par les sources archivistiques qu'elle a suscité, a le mérite pour nous d'éclairer les rapports qui unissent le prince et le sol urbain, révélant conjointement les structures d'un espace et d'un pouvoir.

ESPACE PUBLIC, ESPACE PRIVE : DEFINITIONS ET IMBRICATIONS

Les structures de la propriété immobilière et les normes de l'édification privée restèrent longtemps à Milan hors de portée de l'effort réglementaire de la puissance publique. De ce point de vue, l'État seigneurial des Sforza ne cherchait nullement à innover, reconduisant les antiques dispositions statutaires, rassemblées pour la pre- 
mière fois en 1216 dans le Liber consuetudinum Mediolani ${ }^{1}$, complétées ensuite dans les rédactions successives des statuts communaux $^{2}$. Il s'agissait, très classiquement, de délimiter et de protéger un espace public, dont les normes juridiques garantissaient l'inappropriabilité. L'affirmation du droit public sur les murailles, la voirie, les canaux, les places civiques, inspirent des dispositions et des règlements de police urbaine de plus en plus minutieux, dont la répétition même dit la difficulté à s'imposer. Sur ce sujet, rien à Milan ne s'écarte des grandes lignes d'une histoire générale de l'espace public, bien connue en ce qui concerne les communes italiennes ${ }^{3}$. Mais cet effort a son revers : le travail juridique de la puissance communale vise à délimiter, de plus en plus finement, une portion de l'espace urbain dégagé de la logique d'appropriation privée; du même coup, toute la part du sol qui ne se trouve pas défini comme espace public est livré aux mécanismes d'un marché immobilier en voie de constitution historique ${ }^{4}$. Ainsi, à mesure que se distinguent les catégories juridiques d'espace public et d'espace privé, à mesure même que s'affirme l'emprise juridique du pouvoir seigneurial sur le premier, augmente son impuissance statutaire à réglementer l'édification privée. Une telle contradiction explique en grande partie, au moins du point de vue du droit, la difficulté du pouvoir princier à imposer un droit d'expropriation ${ }^{5}$.

Cette évolution juridique et statutaire a son importance, elle ne rend toutefois pas compte de l'ensemble et de la complexité de la réalité urbaine. Même les historiens du droit en conviennent : pour repérer les changements majeurs qui affectent, à Milan, les rapports entre le prince et le sol urbain, les rédactions successives des statuts

${ }^{1}$ Liber consuetudinum Mediolani, E. Besta et G.L. Barni éd., Milan, 1949. Sur la législation statutaire lombarde en général : A. LATTES, Il dirito consuetudinario delle città lombarde, Milan, 1899.

${ }^{2}$ La réglementation urbanistique milanaise a été étudiée du point de vue statutaire par F. SinATTI D'AMIco, Per una città. Lineamenti di legislazione urbanistica e di politica territoriale nella storia di Milano, Lodi, 1979.

${ }^{3}$ Synthèse commode dans J. HeERs, Les villes d'Italie centrale et l'urbanisme : origines et affirmation d'une politique (environ 1200-1350), dans MEFRM, 101, 1989, 1, p. 67-93. Dans un cadre plus vaste, voir les analyses et les bibliographies de A.I. PINI, Città, comuni e corporazioni nel medioevo italiano, Bologne, 1986.

${ }^{4}$ Sur la question complexe du marché immobilier, et sur la nécessité d'en étudier la construction historique, voir les remarques liminaires de J.-C. MAIRE VIGUEUR, dans D'une ville à l'autre : structures matérielles et organisation de l'espace dans les villes européennes (XIII ${ }^{e}-X V I^{e}$ siècle). Actes du colloque organisé par l'École française de Rome avec le concours de l'Université de Rome (Rome 1'r-4 décembre 1986), Rome, 1989, p. 1-14.

${ }^{5}$ Sur cette question épineuse, voir l'étude juridique de U. Nicolini, La proprietà, il principe e l'espropriazione per pubblica utilità. Studi sulla dottrina giuridica intermedia, Milan, 1952. 
communaux sont d'une moindre utilité que les actes de la pratique ${ }^{6}$. Or, si l'ordre juridique construit des catégories d'espace public et d'espace privé de plus en plus précises, de plus en plus étanches, c'est au contraire la superposition, le chevauchement ou l'imbrication de ces espaces que révèle la lecture des archives milanaises.

Ces archives elles-mêmes, par leur ampleur ou leur densité, dessinent d'ailleurs une cartographie différenciée du sol urbain. Les espaces publics s'imposent au regard de l'historien comme des môles de concentration archivistique. Surdéterminés et surprotégés, ils sont par consćquent amplement documentés et accèdent pour nous à une précision topographique qui s'évanouit progressivement à leurs confins. Et, parmi eux, la place civique semble être l'espace public par excellence, lieu superlatif qui canalise et qui concentre le souci réglementaire. L'ouverture de la place du Broletto dans la capitale lombarde avait, en 1228, commandé un plan global de planification urbaine ${ }^{7}$. Des portes du palais public partaient les six rues principales, structurant fermement l'espace urbain jusqu'aux six portes de l'enceinte. La place civique devenait ainsi le cœur d'un vaste complexe urbanistique, que les seigneurs successifs de Milan eurent grand soin d'orner et d'aménager : ainsi cette place quadrangulaire fut-elle bordée au nord par le Tribunale di Provvisione, à l'est par les prisons du podestat, au sud par la Loggia degli Osii de Matteo Visconti (1316) et par le portique d'Azzone (1336) à la disposition des changeurs. A l'écart de la cathédrale, le Broletto combine donc vie politique et activité commerciale, et cet équilibre crucial exige de la puissance publique une vigilance toujours plus pointilleuse. La circulation des hommes y est par exemple strictement réglementée ${ }^{8}$, et

- Cela est particulièrement vrai pour le $\mathrm{XV}^{\mathrm{e}}$ siècle, où les statuts semblent fossiliser les antiques dispositions juridiques du pouvoir communal, dont les Sforza veulent être les garants, même si la réalité de leur pratique administrative en bafoue les principes. Ainsi, comme l'admet F. SinATTI D'AMIco dans son étude des statuts urbains milanais, «Si deve però sottolineare che nel secolo che va dalla fine del 1390 al Cinquecento molte sono le trasformazioni che si sono verificate più che nella legislazione vera e propria, nella prassi amministrativa che si è maturata nel periodo signorile», op. cit., p. 225. A lire les statuts de 1498, on voit, par exemple, s'affirmer avec une vigueur redoublée l'inaliénabilité de l'usage de l'eau commune, alors qu'au même moment, la crise fiscale de l'État seigneurial l'engage dans une politique systématique d'aliénations de ses droits sur les canaux.

${ }^{7}$ Ce plan nous est connu par le texte de Bernardino Corio, historien milanais de la fin du XVe siècle qui a eu accès à des documents publics aujourd'hui disparus, Storia di Milano, éd A. Morisi Guerra, Turin, 1978, p. 347. À ce sujet, voir G. Soldi Rondinini, Le strutture urbanistiche di Milano durante l'età di Ludovico il Moro, dans Milano nell'età di Ludovico il Moro, t. 1, Milan, 1983, p. 553-576.

${ }^{8}$ Archivio di Stato di Milano (dorénavant noté ASMi), Registri Panigarola, 2, fo $188 \mathrm{v}-189 \mathrm{r}, 29$ avril 1413. Édité par L. Osıo, Documenti diplomatici tratti dagli Archivi milanesi, vol. 2, Milan, 1872, p. 16-17. L'accès au Broletto est interdit aux 
les transactions commerciales contrôlées ${ }^{9}$. La place civique est un espace protégé de tous les empiétements, et jusqu'à celui des puanteurs puisqu'on spécifie en 1421 qu'il est interdit, sous peine de 10 livres d'amendes, d'y faire "fondre ou égoutter du suif, du lard, du saindoux ou d'autres choses du même genre entre les confins du dit Broletto, et d'exercer les métiers ou l'activité susdits desquels naît une odeur intolérable" ${ }^{10}$.

Ces dispositions statutaires ne placent pas pour autant le sol public à l'écart des mécanismes du marché immobilier. En effet, les édifices du Broletto peuvent être, dès la fin du XIVe siècle, concédés à des corporations, voire à des particuliers. En 1399, Gian Galeazzo Visconti concède au Collège des notaires de la ville une partie du palais communal ${ }^{11}$. Plus intéressant : en 1433, Filippo Maria Visconti autorise l'Università dei Mercanti à édifier un bâtiment dans les confinei Broletti, près de l'église S. Michele al gallo, avec une clause restrictive (l'édifice ne doit empiéter sur le sol public que par les pilastres de son portique) ${ }^{12}$. Cette concession se fait en échange de 4 livres de chandelles annuelles : cens recognitif, qui vaut précisément

charrois et aux bestiaux et la place désencombrée "...adeoquod nobiles et mercatores, aliique cives Mediolani inibi pro sui libito libere conversari et morari (sic),..."

${ }^{9}$ Pour la vente du grain par exemple, dispositions du 5 décembre 1437, Archivio storico civico di Milano (dorénavant noté ASCMi), Provisionum 14161450, f $140 \mathrm{v}$.

${ }^{10}$ ASCMi, Provisionum 1416-1450, fo 56, 20 juin 1421. Au-delà de l'aspect anecdotique de cette ségrégation olfactive, ce document a l'avantage de délimiter précisément les confinei Broletti, espace public élargi qui se définit par la proximité des portes du palais public, et jouit ainsi de sa protection statutaire : de la porte du Broletto vers Porta Nuova jusqu'à "l'osteria detta de la Cernia» qui est près la ruelle qui va à l'église $S$. Protaso ad monachos, de la porte du Broletto de Porta Cumana jusqu'à la pharmacie de Giovannolo Sansono dans la contrada da Cordusio, de la porte du Broletto des portes Ticinese et Vercellina dans la contrada delle Armi jusqu'à la pharmacie à l'enseigne de la cloche, et de la porte du Broletto de Porta Romana de la contrada dei Vairari jusqu'à l'endroit où est la draperie des fils de Franceschino de Lomeno, et de la porte de Porta Orientalis jusqu'à la bottega frixarie de: Giacomino de la Curte et de ses frères. Cette description ne permet pas de cartographier précisément la zone, mais elle donne une idée de la représentation de l'espace autour du Broletto, dont les accès prennent le nom des portes de la murailles où ils mènent. Ces grandes voies publiques sont donc bien un élément structurant de l'espace urbain et constituent, avec quelques boutiques ou négoces dont l'énumération témoigne de l'activité de la zone, le repère essentiel dans la reconnaissance de cet espace.

"ASMi, Diplomi e dispacci sovrani (DDS), 1, 30 juillet 1399. Sur le Collège des Notaires milanais, voir A. Livia, Notariato e documento notarile a Milano dall'Alto Medioevo alla fine del Settecento, Rome, 1979.

12 "...non occupando solum illius loci de subtus nisi quatenus expediat pro fundamento pilastrorum et aliorum neccessariorum pro constructione». ASCMi, Litterarum ducalium 1426-1436, Reg. 5, fo 103v-104. 
reconnaissance du droit éminent du propriétaire (ici, la commune) sur le sol à bâtir. On est alors en présence du cas de figure le plus classique de dissociation de la propriété immobilière, à ceci près que le sol qu'elle affecte est un espace public protégé par la législation statutaire.

La concession par la commune de boutiques sous les portiques du Broletto se généralise dans le courant du XVe siècle. Ces concessions ad pensionem deviennent pour elle une source de revenus non négligeable : dès 1404 , le duc de Milan ordonne à l'administration communale de dresscr une liste chiffrée de tous les concessionnaires du Broletto $^{13}$. Ceux-ci prennent en charge les réparations de ces édifices $^{14}$ et les redevances exigées sont soumises à des réévaluations constantes : en 1430, Damiano de Gallinis obtient le renouvellement de son bail de 9 ans pour "une boutique de la commune de Milan, située sous la porte du Broletto, dite porte de Porta Cumana, à gauche en entrant», moyennant un fitto de 14 florins annuels. Or, cette même concession était cédée en 1414 pour 8 florins, et l'on sait également que Pietro, le père du dit Damiano, ne payait à une date indéterminée que 4 florins ${ }^{15}$. Cette concession ne vaut, sauf cas exceptionnels ${ }^{16}$, que pour l'édifice construit et n'implique aucun droit sur le sol, qui demeure propriété communale ${ }^{17}$.

Entre l'espace public et l'espace privé, les délimitations ne sont donc pas aussi étanches que la lecture des statuts communaux pourrait le laisser croire, et la pratique généralisée de la concession ad fictum induit des formes complexes et variées de dissociation de la propriété. Le cas du Broletto est, à cet égard, exemplaire, mais l'analyse vaudrait également pour la concession de portions d'enceintes, ou de débarcadères (soste) au fil des canaux circulaires. En tous cas,

${ }_{13}^{13}$ ASCMi, Sindicorum 1395-1409, Reg. 1, fo 128v, 2 mars 1404.

${ }^{14}$ Ainsi, par exemple, le banquier Albrigolo Capono, qui paye 2 florins par an un banc près d'une porte du Broletto , ASCMi, Sindicorum 1395-1409, Reg. 1, fo 143,16 mars 1405 .

${ }_{15}^{15}$ ASCMi, Litterarum ducalium 1426-1436, Reg. 5, fo 65-66r, 22 mai 1430.

${ }^{16}$ En 1461, Francesco Sforza concède à Gaspare de Vicomercato, condottiere ducal, la moitié d'un banc près du dernier pilastre du palais du Broletto, donnant vers la porta Nuova, en pleine propriété du sol, "de pleno jure». La personnalité exceptionnelle du concessionnaire (récompensée ici "pour sa fidélité») explique cette entorse à la règle commune, présentée comme telle dans le document. ASCMi, Litterarum ducalium 1456-1461, Reg. 11, fo 261r-262r, 2 juillet 1461.

${ }_{17}$ De nombreux cxcmples de ces concessions se trouvent dans les registres des Litterarum ducalium de l'ASCMi, ainsi que dans le fonds Località milanesi, cart. 214, pour les années 1449, 1450,1451, 1457, 1458, 1479, 1481, 1482, 1485, $1486,1487,1488,1492,1494,1496,1498,1499$. En 1498, les revenus que la commune tire de ces concessions s'élèvent à 936 livres, ASCMi, Litterarum ducalium 1497-1502, Reg. 16, fo 41, 16 février 1498. 
les formes d'imbrication entre l'espace public et l'espace privé constitue un des enjeux essentiels de la politique immobilière du pouvoir princier. Cette problématique a été depuis longtemps abordée par la question des empiétements. La lutte contre l'usurpation privée (travaux abusifs, encombrements des places, débordements sur les voies publiques, comblement des canaux, etc.) alimente une activité normative précoce ${ }^{18}$. Les registres des magistratures spécialisées dans la police des rues ayant disparus, cette question est essentiellement documentée à Milan par des lettres de grâce ducale revenant sur certaines condamnations ${ }^{19}$. Dans la seconde moitié du XVe siècle, une autre série de documents éclaire cette question : il s'agit des licentie edificandi concédées par le pouvoir ducal à des particuliers qui veulent bâtir des édifices empiétant, tout ou partie, sur l'espace public ${ }^{20}$. Sur 39 occurrences relevées de 1450 à 1484, ces licences autorisent l'édification de murs dans 13 cas, d'abris, de baraques ou d'étal dans 5 cas, de balcons et de fenêtres dans 3 cas. Le reste (soit 18 licences) concernent des portiques. De façon très significative, la concession porte précisément sur le nombre de colonnes ou de pilastres : ainsi, à titre d'exemple, Battista de Galiate est autorisée à "planter trois colonnes à Porta Nuova, paroisse San Bartolomeo sopra la Cantarana, entre Borgo Spesso et Borgo de Ranchate, près de sa boutique» moyennant 7 livres impériales ${ }^{21}$. Les prix de la concession varient selon le quartier

${ }^{18}$ Pour une approche synthétique de cette question très classique, voir, par exemple, J. Heers, La ville au Moyen Âge en Occident. Paysages, pouvoirs et conflits, Paris, 1990, p. 353 sq.

${ }^{19}$ Ces lettres se trouvent aujourd'hui dans ce qui reste des archives de l'Ufficio dei sindaci de Milan, ASCMi, Sindicorum 1395-1408, Reg. 1. Pour une présentation de ce registre, voir C. SANTORo, I registri dell'Ufficio di Provvisione e dell'Ufficio dei sindaci (Inventari e regesti dell'archivio civico, vol. 1), Milan, 1929, p. XIX sq. A titre d'exemple, la duchesse de Milan annule en 1408 une condamnation infligée par le Giudice delle strade aux héritiers de Compadre Sado à cause d'un pilastre et d'une haie débordant sur la voie publique. Après enquête, il s'avère que le terrain sur lequel ils empiètent n'est pas un terrain communal, mais un fonds de l'église de Santa Maria alla Porta., op. cit., fo 185, 6 décembre 1408.

${ }^{20}$ ASCMi, Litterarum ducalium 1450-1487, Reg. 10. La dénomination de ce registre est très certainement fautive : l'analyse des actes qu'il contient (qui ne sont pas seulement des lettres ducales) le rapproche du Sindicorum 1395-1408 précédemment cité. Nous croyons que selon toute vraissemblance, il constitue le second registre conservé de l'Ufficio dei Sindaci. Voir, pour une présentation sommaire de ce registre, C. SANTORO, I registri delle lettere ducali (inventari $e$ regesti dell'archivio storico civico, vol. 2), Milan, 1961. Pour ce qui nous intéresse, ce registre contient 39 licences d'édification, de 1450 à 1484 .

${ }^{21}$ ASCMi, Litterarum ducalium 1450-1487, Reg. 10, fo 157r., 23 février 1480. La concession porte sur deux colonnes ou pilastres dans 11 cas, sur trois dans 5 cas et sur quatre dans 2 cas. 
et la proximité des grands édifices publics, le nombre des colonnes et pilastres, et leurs matériaux : plus chers que ceux en bois, les pilastres de pierre voient leurs dimensions réglementées ${ }^{22}$. Ces normes méticuleuses ne sont pas sans intérêt; elles prouvent que ce qui est précisément taxé dans le portique ou l'étal de la boutique, ce sont leurs points d'appuis "sur le sol de la commune», comme le précisent nos documents. De même, c'est lorsqu'ils jouxtent l'espace public (canaux, murailles, voirie) que les édifices privés sont soumis à autorisation préalable. On l'aura compris : l'effort réglementaire en direction des éléments saillants de l'ćdification privée ne doit pas seulement se comprendre dans une logique de lutte contre les accaparements, mais également - et peut-être surtout - comme un moyen détourné d'imposer un contrôle sur la construction privée. À Venise, le Piovego tente pareillement, à partir notamment des portiques et des terrasses hautes (altana) de "faire entrer dans les faits le permis de construire ${ }^{23}$.

Le pouvoir princier ne se cantonne pas dans une politique défensive, et il ne faudrait pas, à partir des dispositions inlassablement répétées des statuts urbains, imaginer l'espace public comme perpétuellement assiégé par les appétits de l'édification privée. Entre l'un et l'autre, au contraire, s'établissent des rapports complexes d'imbrication et d'enchevêtrement, commandés par des concessions qui, souvent, créent des formes dissociées de propriété. L'espace public, enfin, constitue pour le pouvoir princier un point d'appui qui lui permet, de proche en proche, d'imposer par la pratique administrative des mécanismes de contrôle du marché immobilier.

LES LETTRES DE CONCESSIONS DUCALES : UNE TENTATIVE PRINCIÈRE DE CONTRÓLE DU MARCHÉ IMMOBILIER

Au moment où Francesco Sforza tente d'imposer ses licences d'édification, un autre type de concessions ducales, plus difficiles à définir juridiquement, fait son apparition dans les registres ${ }^{24}$. Il s'agit de "licentiam procedendi ad emphiteoticam locationem». Ces lettres réglementent l'accès à la propriété pleine ou partielle de bien-

${ }^{22}$ Ibid., fo 112v, 4 mai 1460, devant une boutique de draps, deux pilastres de pierre "larges de 15 onces et longs de 13 ".

${ }^{23}$ E. CROuzet-Pavan, "Sopra le acque salse". Espaces, pouvoir et société à Venise à la fin du Moyen Âge, t. 1, Rome, 1992, p. 231 sq.

${ }^{24}$ Dans le registre de l'ASCMi, Litterarum ducalium 1456-1461, Reg. 11, ces lettres de concessions ducales forment 63 actes sur 272 , soit près du quart de l'ensemble. C'est essentiellement durant cette première décennie de la seigneurie de Francesco Sforza qu'enfle la masse de ces concessions. 
fonds, dans le contado pour les deux tiers, et dans la ville de Milan pour le dernier tiers. Pour ce qu'elles révèlent des structures de la propriété milanaise et de l'intérêt qu'y porte le pouvoir princier, elles valent sans doute la peine qu'on s'y attarde.

La concession ducale est, dans tous les cas, la réponse à une supplique, intégralement ou en partie citée dans la narratio du document («Recepimus supplicationem tenoris proxime subsequentis...»). C'est le bailleur d'un fonds qui fait la requête auprès de la chancellerie ducale. Ce bailleur est presque toujours un des ordres religieux qui, à Milan comme ailleurs, furent et sont encore au $X^{e}$ siècle les principaux acteurs du marché immobilier urbain. La requête est souvent appuyée par le vicaire de la cour archiépiscopale, toujours par le conseil de justice, et l'Ufficio di Provvisione, qui est l'organe central de l'administration communale ${ }^{25}$. Parfois, l'avis d'un ingénieur de la commune est sollicité. Ainsi, le circuit de la requête et de la recommandation peut être reconstitué. La licence ducale porte donc sur la concession ou la location d'un bien-fonds ${ }^{26}$. Les édifices sont décrits brièvement, selon les normes notariales : "cameris cum solario, cameris cum terra, pertico, curte, apertus versus zardinum ${ }^{27}$ sans que jamais l'on puisse avoir une idée précise de leur superficie. En revanche, ils sont situés, par portes et paroisses («Parochia San Nicola, Porta Vercellina» pour l'exemple précédent), et la proximité avec les voies publiques ou les canaux est fréquemment signalée. Le locataire ou le concessionnaire du bien-fonds est toujours cité, et parfois même, il s'associe à la requête. Il fait souvent partie de cette notabilité milanaise qui peuple alors l'appareil d'État dont Francesco Sforza tente de s'assurer la fidélité. On y reconnaît même certaines des personnalités les plus en vue comme Daniele de Marliano ${ }^{28}$ ou le comte Gaspare de Vicomercato, condottiere ducal et mécène fastueux, promoteur

${ }^{25}$ Sur les institutions communales milanaises, voir A. CоLомво, L'amministrazione civile di Milano comunale, dans Archivio storico lombardo, LXXXVII, 1960, p. 267-293.

${ }^{26}$ Pour désigner ce type de locatio-conductio, nos documents utilisent le plus fréquemment les termes de locationem emphiteuticam. La rente (fitto, livello, ou fitto livellario) exigée par le bailleur comporte un cens annuel extrêmement variable, de 5 à 40 florins annuels, souvent payables en deux fois, mais ni droit d'entrée, ni droit de renouvellement, ni droit de mutation. Quant à la durée du bail, elle n'est que très rarement signalée. Sur les clauses des contrats emphytćotiques, voir P. TOUBERT, Emphyteusis, Erbleihe, dans Lexikon des Mittelalters, Bd. III, Munich-Zurich, 1986, col.1892-1895. 1458.

${ }^{27}$ ASCMi, Litterarum ducalium 1456-1461, Reg. II, fo 124v-126r, 18 février ${ }^{28}$ Ibid., fo $83 \mathrm{v}-85 \mathrm{r}, 10$ mai 1457. 
de la construction de l'église de Santa Maria delle Grazie ${ }^{29}$. Certains nous sont connus par leur carrière podestataire, comme Giovanni Giacomo de Madregnano, d'autres appartiennent au conseil secret, tel Guarnerio de Castiliono ${ }^{30}$.

C'est donc au moment d'une mutation, ou d'un renouvellement de bail, que le propriétaire demande, pour un bien-fonds donné et un preneur cité, une concession ducale. Celle-ci ne peut donc tenir lieu d'acte notarié, elle en est le préalable juridique : "Concedimus per praesentes ac dispensamus et decernimus, quod ad locationem emphiteoticam, de qua super, procedi possit cum et sub pactis et conventionibus superius expressis ${ }^{31}$. Enfin, cette concession est parfois assortie de conditions, portant sur la valeur de la rente, la modification du régime de propriété, l'obligation de réparations ou de travaux divers ${ }^{32}$.

A l'issue de cette brève description formelle, une question essentielle demeure : qu'est-ce qui justifie la nécessité, pour un bailleur de bien-fonds, d'une concession ducale? Autrement dit, quel droit concède réellement le duc en s'insinuant dans une transaction privée? Comme on l'a vu, une telle exigence princière est légitime lorsque le bien-fonds jouxte un espace public, ou concerne d'une manière ou d'une autre le bien commun que le pouvoir ducal doit défendre et protéger : ainsi, la proximité d'une voie d'eau ou de la voirie publique peut être signalée dans les lettres de concessions ducales, mais elle est loin de constituer la règle. Nous sommes ici dans un autre cas de figure, qui n'est évidemment pas prévu par la législation réglementaire. Les lettres de concessions ducales se rapprochent formellement de la licentia pro bonis vendendis, qui concerne normalement des biens sur lesquels la chambre ducale possède des droits ${ }^{33}$. En exigeant une telle licence pour une tran-

${ }^{29}$ Ibid., fo 261r-262r, 2 juillet 1461, et Litterarum ducalium 1462-1472, Reg. 12, fo 12r-13r, 6 mai 1458.

${ }_{30}$ Ibid., fo 64v-66V, 20 octobre 1456. Il a servi successivement Filippo Maria Visconti, la république Ambrosienne, et Francesco Sforza qui le place au Conseil secret le 11 mars 1450. Il est ambassadeur à Venise en 1454, lors de la ratification de la paix de Lodi. Voir C. SANTORo, Gli Uffici del dominio sforzesco, Milan, 1947, p. 3 sq.

${ }^{31}$ Annali della Fabbrica del Duomo di Milano, éd. C. Cantù, 7 vol., Milan, 1877-1880, vol. 2, p. 199, $1^{\text {er }}$ mars 1460.

${ }^{32}$ Ainsi, le couvent San Vincenzo est-il autorisé en 1489 à concéder une de ses maisons à Battista Negri, à condition que soient murées toutes les fenêtres qui donnent sur le cloître, le réfectoire et le dortoir des sœurs (ASMi, DDS, 8, $n^{\circ} 23,24$ juillet 1489).

${ }_{33}$ Voir A.R. NATALE, Stilus cancellariae. Formulario visconteo-sforzesco (Acta italica, 19), Milan, 1979, p. CXVI sq. pour l'analyse diplomatique de la licence ducale, et doc. 141, p. 178-179, pour le formulaire d'une licentia pro bonis vendendis. 
saction privée, Francesco Sforza cherche à étendre son contrôle à l'ensemble des mutations qui animent le sol urbain, sans que les conditions précises de ce contrôle ne soient jamais statutairement définies - ce qui confirme, s'il en était besoin, le fait que la pratique administrative du pouvoir princier tend à s'écarter des règles normatives édictées par l'autorité communale.

Quelles sont les raisons de ce "coup de force» juridique? Ce que tente le pouvoir princier, c'est d'abord sans doute de contrôler le marché immobilier, en limitant notamment la hausse des rentes, puisque la concession ducale peut se lire simplement comme une autorisation de réévaluation du "fitto annuo". Si on devine partout cette hausse, il est très difficile de la mesurer. Les lettres comportent souvent deux données : la rente obtenue jusqu'alors, et celle que souhaite désormais le propriétaire. Entre les deux, l'augmentation varie généralement de 20 à $30 \%$, mais il est difficile d'en dire plus, dans la mesure où l'on sait seulement que la rente précédente était perçue «in antico». Un seul indice nous donne une indication supplémentaire : l'Hôpital San Martino baille en 1456 un bien-fonds avec une maison attenante à la voie publique à Lanfranco de Inzago pour 5 florins ${ }^{34}$. En 1459, le même bien-fonds est baillé à Ambrogio de Garbagnate, mais pour 6 florins, ce qui représente une augmentation de $20 \%$ en 3 ans $^{35}$. Cette tendance ne doit pas nous étonner, compte tenu de la conjoncture démographique que connaît alors la capitale lombarde. Relativement épargnée par la peste noire, elle avait essuyé de plcin fouet les épidémies de 1398-1401, 1422-1425 et 1437-1439. Mais, selon les travaux de Giuliana Albini, c'est bien en 1450 que Milan connaît l'onde de choc la plus meurtrière (peut-être 30.000 morts) ${ }^{36}$. Avec la paix de Lodi en 1454 s'ouvre une longue période de reprise démographique, assortie d'une extrême mobilité de population. Le pouvoir ducal favorise d'ailleurs cette repopulation de la capitale lombarde, en accordant massivement des octrois de citoyenneté et des exemptions fiscales ${ }^{37}$. Tout cela alimente une

${ }^{34}$ ASCMi, Litterarum ducalium 1456-1461, Reg. 11, fo 17v-19r, 22 avril 1456.

${ }^{35}$ Ibid., fo $182 \mathrm{r}-183 \mathrm{v}, 26$ juin 1459.

${ }^{36} \mathrm{G}$. AlBINI, Guerra, fame, peste. Crisi di mortalità e sistema sanitario nella Lombardia tardomedioevale, Bologne, 1982, notamment p. 22 sq. Voir également, du même auteur, La mortalità in un grande centro urbano nel '400: il caso di Milano, dans Strutture familiari, epidemie, emigrazione nell'Italia medievale, éd. R. Comba, G. Piccinni et G. Pinto, Naples, 1984, p. 117-133.

${ }^{37}$ On a conservé, de 1450 à 1455 , soixante-dix concessions de citoyenneté. Cf. G. Albini, Guerra, fame, peste, op. cit., p. 29. De même, en janvier 1452, un décret annule les dettes des "artesani, sì lavoranti como magistri de botteghe" exilés de Milan pour faciliter leur retour, Codice visconteo-sforzesco, ossia Raccolta di leggi, decreti e lettere famigliari dei duchi di Milano, éd. C. Morbio, Milan, 1846, p. 343. 
forte demande de logements, et donc une spéculation qui tire certainement le marché à la hausse ${ }^{38}$.

Dans cette conjoncture de reprise, très favorable aux propriétaires, on comprend mieux que le pouvoir ducal tente de freiner la hausse des rentes et des loyers. D'abord peut-être, parce qu'engagé dans une ambitieuse politique de grands travaux, et en l'absence d'un droit établi d'expropriation pour utilité publique, le prince est le premier client du marché immobilier ${ }^{39}$. Ensuite, parce qu'il poursuit une politique démographique inaugurée par Gian Galeazzo Visconti en 1386-1389, qui consiste, comme l'a montré Amintore Fanfani, à fixer une main-d'œuvre artisanale dans les quartiers industrieux de la ville ${ }^{40}$. Pour conserver son pouvoir d'attraction, Milan doit proposer des logements à prix raisonnable. Le duc tente donc de peser sur les prix, directement par ses lettres de concession, indirectement lorsqu'il cherche à limiter la concentration immobilière. Ainsi, les concessions de citoyenneté sont-elles assorties du droit d'acheter des biens en ville. Mais ce droit est toujours limité, généralement à 500 florins dans les années 1450 , 1000 dans les années 1460 - le relèvement du plafond trahissant d'ailleurs la difficulté politique qu'il y avait à tenter de contrarier cette logique de concentration foncière.

Cette difficulté traduit toutefois une contradiction plus profonde. Après la mort de Francesco Sforza, les lettres de concessions ducales se raréfient, disparaissant même des registres pendant plusieurs années. C'est là sans doute un aveu d'échec pour cette tentative audacieuse, mais désordonnée. Le pouvoir princier n'avait sans doute aucune chance de contrarier une hausse de la rente mue par la vigueur du marché immobilier. De plus, son ambition réglementaire, fondée sur une pratique administrative et non sur sa formalisation juridique, achoppait sur une contradiction socio-politique essentielle : comment (et pourquoi?) promouvoir une politique de stabilisation de la rente alors que la dynamique du marché immobilier enrichit notables, fidèles et officiers de l'État seigneurial qui savent s'intercaler entre propriétaires et locataires?

${ }^{38}$ Pareil phénomène se vérifie, par exemple, à Paris, pour les années 1460. Voir S. Roux, Le coût du logement ordinaire à Paris au XVe siècle, dans D'une ville à l'autre, op. cit., p. 243-263.

${ }^{39}$ Sur la politique édilitaire de Francesco Sforza, nous nous permettons de renvoyer à $\mathrm{P}$. BOUCHERON, Les expressions monumentales du pouvoir princier à Milan au temps de Francesco Sforza (1450-1466), dans Les princes et le pouvoir au Moyen Âge. Actes du XXIII Congrès des historiens médiévistes de l'enseignement supérieur (Brest, 1993), Paris, 1993, p. 117-135.

${ }^{40}$ A. Fanfani, Aspetti demografici della politica economica del ducato di Milano, dans Saggi di storia economica italiana, Milan, 1936, p. 123-157. 


\section{LE POUVOIR PRINCIER FACE AUX FORMES DISSOCIÉES DE LA PROPRIÉTÉ}

Dans sa tentative de contrôle politique du marché immobilier, le pouvoir princier ne se heurtait pas seulement au mouvement de la rente, mais à la dynamique de complexification des régimes de propriété. Pendant une décennie (la période cruciale 1455-1465), les lettres de concessions ducales jettent donc un coup d'éclairage inattendu sur les formes et les mécanismes de dissociation de la propriété. À ce sujet, un point doit être éclairci : les concessions portent-elles seulement sur le sol, ou sur l'ensemble du bâti? Le 18 janvier 1463, le monastère de Brera concède aux héritiers des frères De Fayno des biens-fonds pour une somme minime : 5 sous pour un terrain Porta Nuova, 10 sous, 6 deniers et une livre de cire pour un autre, Porta Cumana ${ }^{41}$. Ici, pas de doute : les concessionnaires ne payent que la reconnaissance du droit éminent du monastère sur un terrain à bâtir. Mais ailleurs, lorsque le cens ou le loyer demeure minime, on peut hésiter : ainsi une petite maison est louée pour 32 sous $^{42}$ alors qu'un terrain se baille pour 29 sous $^{43}$.

Toutefois, il apparaît clairement que dans la plupart des cas, les institutions ecclésiastiques louent ou concèdent la proprietas et la superficies, ou plus exactement, pour reprendre le vocabulaire de nos documents, "uno sedimine cum edifitiis». Le mot doit nous arrêter un instant, comme fossile d'une évolution historique largement achevée au $\mathrm{XV}^{\mathrm{e}}$ siècle. Sedimine, ou plus rarement sedime, dérive évidemment du latin sedimen, quc Du Cange définit comme «locus quiuis vacuus, idoneus ad aedificandum uel plantandum». Le mot est attesté à Milan dès le $\mathrm{IX}^{\mathrm{e}}$ siècle : en 812 , un «negotians de Mediolano" acquiert une "casa» et un "sedimen" d'un vassal du roi $^{44}$. Sedimen a alors cette signification précise de terrain à bâtir, terrae vacantes ad domos faciendas, et s'oppose donc à edificia. L'expression "sedimine cum edifitiis" est à la fois paradoxale et remarquable, puisqu'elle témoigne de l'ancienne dissociation juridique de la propriété résultant de la politique de lotissements menée par les ordres religieux.

Une autre trace de dissociation juridique peut se trouver dans la constitution même des rentes exigées par le propriétaire. A la somme en florins ou en lires impériales, qui constitue le véritable rendement du patrimoine immobilier, s'ajoute souvent un cens en

\footnotetext{
4! ASCMi, Litterarum ducalium 1462-1472, Reg. 12, fo 27v-29v.

${ }^{42}$ ASCMi, Litterarum ducalium 1456-1461, Reg. 11, fo 182r-183v, 26 juin 1459.

${ }^{43}$ ASCMi, Litterarum ducalium 1462-1472, Reg. 12, fo 27v, 18 janvier 1463.

${ }^{44}$ Codex diplomaticus Langobardiae, $M H P, 1873, \mathrm{n}^{\circ} 87$. Voir, sur ce point, $\mathrm{F}$. SINATTI D'Amico, op. cit., p. 111 sq.
} 
nature : 5 florins et deux chapons pour un bien-fonds avec maison Porta Nuova en $1456^{45}, 7$ florins et un œuf pour un fonds avec cour, puits et jardin Porta Ticinese ${ }^{46}$, ou, plus surprenant, 12 florins et 5 brassées de futaine, 1 livre de coton et 4 onces de fil que le monastère San Pietro Celestino exige de son bailleur pour une maison de la paroisse San Babila ${ }^{47}$. Il s'agit sans doute, au moins dans les deux premiers cas cités, d'un cens recognitif, portant reconnaissance du droit éminent du propriétaire sur le sol urbain. Ainsi, la dissociation juridique de la propriété urbaine n'existe plus qu'à l'état de trace fossile, puisque enfouies dans le vocabulaire de la concession comme dans la constitution de la rente, on peut encore distinguer deux strates, portant l'une sur le sol et l'autre sur l'immeuble.

Le concessionnaire des biens immobiliers n'en est pas moins qu'un propriétaire partiel. Du point de vue des droits et des usages, d'abord. Qui, du bailleur ou du preneur, doit prendre en charge les réparations? On pourrait penser que les petits travaux, seuls, incombent aux concessionnaires. Ainsi, nombre de propriétaires justifient leur augmentation de rente auprès du pouvoir ducal par l'ampleur des améliorations qu'ils ont du supporter, améliorations qui touchent parfois au bien public. Le recteur du monastère de Sant'Ambrogio argue du fait qu'il a supporté le financement de la réparation d'un pont, dit de la Museta ${ }^{48}$. Pourtant, il semble bien que certaines réparations d'ampleur soient à la charge du preneur, et c'est même la concession ducale qui en fixe le montant. En février 1458 , le Comte Gaspare de Vicomercato est autorisé à prendre ad livello deux terrains avec édifices Porta Vercellina, pourvu qu'il y fasse 300 florins de réparations ${ }^{49}$. De même, Matroniano de Braschis, autre grand personnage de l'oligarchie milanaise, quand il prend en concession des immeubles de rapport dans le quartier industrieux de San Eufemia : le duc exige de lui 500 livres impériales de réparation en six ans, et ce après avoir pris l'avis de Giovanni della Porta, ingénieur de la commune ${ }^{50}$. Il s'agit là d'investissements considérables, et si les preneurs s'acquittent réellement de ces obligations, on imagine aisément qu'ils puissent se considérer comme quasi-propriétaires. Une requête originale permet en effet de le penser, celle des fils de Stefano, Donato et Romano de Fayno en 1463. Les trois frères étaient concessionnaires, depuis un temps

${ }^{45}$ ASCMi, Litterarum ducalium 1456-1461, Reg. 11, fo 17v-19r, 22 avril 1456.

${ }^{46} \mathrm{Ibid}$., fo 222v-223r, 20 janvier 1458.

${ }^{47} \mathrm{Ibid}$., fo $217 \mathrm{v}-218 \mathrm{v}, 14$ février 1460.

${ }^{48}$ Ibid., fo 83v-85r, 10 mai 1457.

${ }^{49}$ Ibid., fo $124 \mathrm{v}-126 \mathrm{r}, 18$ février 1458.

${ }^{50} \mathrm{Ibid}$., fo $120 \mathrm{r}-121 \mathrm{v}, 16$ septembre 1455. 
indéterminé, d'un bien-fonds de la Porta Nuova, baillé par le monastère de Brera. Ils assurent qu'au début du bail, celui-ci était «détruit et avec peu d'édifices». Les frères de Fayno y ont fait beaucoup de travaux, qu'un expert, ami commun des preneurs et du propriétaire, Eusebiis de Roxate, "ingegnere e maestro da muro", estime à 850 florins. Fort de cette expertise, et désireux sans doute de rompre l'indivision, les héritiers exigent et obtiennent la permutation de ces biens avec 5 autres bien-fonds répartis dans plusieurs paroisses de Milan, et ce toujours pour la même rente de 23 florins $^{51}$.

D'une certaine façon, les preneurs peuvent donc disposer librement de ces biens, qui sont ainsi soumis à ventes, partages successoraux et aliénations partielles. Les patrimoines ecclésiastiques sont donc engagés dans une dynamique de marché immobilier qui contribue à complexifier les régimes de propriété. Il n'est évidemment pas rare de trouver deux preneurs pour un seul bien-fonds, comme Guarnerio de Castiliono, du conseil secret, et Cristoforo Paliario pour un fonds attenant au canal Nirone en $1456^{52}$, ou parfois un seul preneur pour deux bailleurs, comme l'Hôpital San Martino et l'église Santa Maria al circolo pour ce fonds concédé 9 livres 10 sous à Giovanni de Varisio en $1460^{53}$. La propriété partielle domine donc, et elle dissocie physiquement les biens. Ainsi, en 1458, Santa Maria del Carmine concède quelques pièces d'une maison à deux bailleurs, un particulier (Franceschino de Platis) et une institution (la Fabrique du Dôme) pour 8 livres. Il s'agit d'une cave, d'une chambre et d'un grenier, avec l'usage de l'eau du puits voisin ${ }^{54}$. Cette forme très ténuc de dissociation immobilière impose en effet des règles strictes d'usage des parties communes, de circulation, de droits de passage, etc...

C'est donc sans conteste la propriété partielle qui domine à Milan au XVe siècle, et ce d'autant plus que des éléments supplémentaires de complexité nous échappent. Il s'agit avant tout de la pratique de la sous-location, que l'on devine souvent, sans jamais pouvoir réellement l'appréhender. Conventions orales - comme dans les exemples florentins étudiés par Armando Sapori ${ }^{55}$ - ou contrats conservés par les locataires, les locations à brève échéance

${ }^{51}$ ASCMi, Litterarum ducalium 1462-1474, Reg. 12, fo 27v-29v, 19 janvier 1463.

${ }^{52}$ ASCMi, Litterarum ducalium 1456-1461, Reg. 11, fo 64v-66v, 14 février 1457.

${ }^{53}$ Ibid., fo $224 \mathrm{v}-225 \mathrm{r}, 5$ juin 1460.

${ }^{54}$ Ibid., fo $117 \mathrm{r}-118 \mathrm{v}, 19$ janvier 1458.

${ }^{55}$ A. SAPORI, Case e botteghe a Firenze nel Trecento. La rendita della proprietà fondiaria, dans Studi di storia economica (secoli XIII-XIV-XV), $3^{\mathrm{c}}$ éd., Florence, 1955 , t. 1, p. 305-352. 
ne laissent en effet aucune trace archivistique. Silence logique, tant il conforte l'intérêt des bailleurs. La plupart des grands personnages qui apparaissent dans les lettres de concessions ducales sont euxmêmes des intermédiaires entre les propriétaires et les locataires. On sait combien il peut être rentable de loger des artisans, fussent-ils de médiocre condition, dans les immeubles de rapport des villes de la fin du Moyen Âge ${ }^{56}$. Reprenons un instant la cas de ce Matroniano de Braschis qui obtient en 1455 du monastère de Morimondo un fonds à San Eufemia ${ }^{57}$. La lettre de concession ducale évoque explicitement des «masures en mauvais état pour loger des familles pauvres» et exigent donc, en sus de la rente de 80 livres impériales, 500 livres de réparation en six ans. Cela représente donc, sur six ans, un investissement de 980 livres, dont le preneur doit attendre un rendement élevé. Cette attente ne fut certainement pas déçue, puisqu'on retrouve Matroniano en 1461, six ans plus tard exactement, parmi les XII dell'Ufficio di Provvisione, c'est-à-dire au cœur de l'administration communale ${ }^{58}$, comme si l'entreprise de lotissement populaire était une des voies de l'ascension sociale. La pratique de la sous-location constitue sans doute une des clefs du système : de même qu'à la campagne, si l'on en croit Cipolla, les fictabiles ont dépossédé les établissements religieux de leur propriété immobilière ${ }^{59}$, de même en ville, ce sont les quasi-propriétaires qui prennent en bail le patrimoine foncier de ces institutions et en tirent l'essentiel des bénéfices.

La dynamique du marché immobilier engendre une différenciation sociale accrue, qui produit elle-même des ségrégations plus marquées, tant dans l'espace urbain que dans les régimes de propriété. L'achat d'une maison est évidemment le moyen le plus radical de se protéger des hausses de rentes, mais lorsque ces achats se font progressivement, cette progressivité crée des dissociations supplémentaires. Ainsi, Ambrogio de Gera loue-t-il en 1458 au Monastero Maggiore la moitié de la maison qu'il habite Porta

${ }^{56}$ Des habitations rémoises "louées à menus hostes» aux "camerae" de Besançon, voir, pour les villes françaises, les remarques de J.-P. LEGUAY, La propriété et le marché de l'immobilier à la fin du Moyen Âge dans le royaume de France et dans les grands fiefs periphériques, dans D'une ville à l'autre..., op. cit., p. 135-199, et particulièrement p. 162 sq.

${ }^{57}$ ASCMi, Litterarum ducalium 1456-1461, Reg. 11, fo 120r-121V, 16 septembre 1455.

${ }^{58}$ ASCMi, Litterarum ducalium 1450-1487, Reg. 10, fo 120r, 11 août 1461.

${ }^{59}$ C.M. Cipolla, Une crise ignorée : comment s'est perdue la propriété ecclésiastique dans l'Italie du Nord entre le XIe et le XVI siècles, dans Annales, II, 1947, p. 317-327. Thèses discutées dans G. ChITtolinI, Un problema aperto : la crisi della proprietà ecclesiastica fra Quattrocento e Cinquecento, dans Rivista storica italiana, LXXXXV, 1973, p. 353-393. 
Romana, l'autre moitié lui appartenant en propre ${ }^{60}$. Ln autre, la même année, est propriétaire des deux tiers de son bien ${ }^{61}$. Quant à Andrea de Tadoni, c'est pièce par pièce qu'il a acheté sa maison à la Fabrique du Dôme ${ }^{62}$. Le 21 janvier 1448, il achète 40 florins la dernière chambre de sa maison, qu'il louait pour 3 livres (cela représentait donc un rapport de 4,5\%). Mais tous n'ont pas les moyens financiers de ces patientes conquêtes, et nombreux sont ceux qui, au contraire, perdent pied. On voit ainsi, en 1457, un orfèvre, Donato de Castiliono endetté auprès d'un Gênois à hauteur de 220 ducats d'or, et obligé de lui vendre une maison Porta Orientale sur laquelle il continue pourtant de payer un fitto de 20 florins $^{63}$. La même année, c'est un coutelier qui doit vendre sa maison, constituant pourtant le dernier gage des biens dotaux de sa femme. Après s'être assuré que celle-ci était consentante à la vente, le duc l'autorise, en nommant Martino Porono, principal débiteur du coutelier, fideiussore de la transaction ${ }^{64}$. Les fils ténus de l'endettement participent donc à l'histoire de la dissociation de la propriété.

Celle-ci met en tout cas la puissance publique face à de nouveaux défis. La question fiscale, sur laquelle nous sommes très mal renseignés, doit être déterminante. On sait en effet que l'estimo avait le plus grand mal à démêler l'écheveau touffu des formes dissociées de la propriété, et que cette latence profitait sans doute aux concessionnaires. De ce point de vue, il est donc logique que le duc de Milan profite des lettres de concessions pour débroussailler les régimes de propriété, exigeant quand il le peut de libérer un bienfonds des différentes charges qui le grèvent. D'une façon générale, la multiplication des intermédiaires entre le propriétaire et l'habitant réel d'une maison rend plus aléatoire le contrôle social et l'efficacité de l'action administrative. Un exemple éclairant concerne le financement des travaux d'entretien de la voirie. Celui-ci était à la charge de ses usagers, par tronçons (fagie) selon une répartition précise. Dans la compartizione delle fagie compilée en 1345 sur l'ordre des seigneurs Giovanni et Luchino Visconti, chaque communauté, ordres religieux ou particuliers se voyaient assignés la charge d'entretien d'une partie de voie publique dans les faubourgs de la ville ${ }^{65}$. Tout au 1458 .

${ }^{60}$ ASCMi, Litterarum ducalium 1456-1461, Reg. 10, fo 123v-124r, 5 janvier

${ }^{61}$ Ibid., fo 174r-175r, 7 septembre 1458.

${ }^{62}$ Annali della Fabbrica..., op. cit., t. 2, p. 113.

${ }^{63}$ ASCMi, Litterarum ducalium 1456-1461, Reg. 10, fo 92v, 7 mai 1457.

${ }^{64} \mathrm{Ibid}$., fo 103r-105r, 20 août 1457.

${ }^{65} \mathrm{La}$ compartizione delle fagie est publiée dans Statuti delle strade e delle acque del contado di Milano fatti nel 1346, éd. G. Porro Lambertenghi dans Miscellanea di Storia Italiana, VII, Turin, 1969, p. 311-373. F. Sinatri D'Amico, op. cit., 
long du $\mathrm{XV}^{\mathrm{e}}$ siècle, ce système de répartition continue à être utilisé, même si l'évolution socio-topographique de ces quartiers suburbains oblige à de fréquents réajustements. Dans la seconde moitié du Quattrocento, toutefois, l'essor de l'emphytéose à courte échéance perturbe ce système de répartition : c'est pour trancher une "controverse" entre propriétaires et concessionnaires que le pouvoir ducal ordonne en 1470 qu'un tiers de la somme prévue par la Compartizione soit à la charge des "patrons directs», et deux tiers à la charge de ceux qui détiennent les bâtiments in affito ${ }^{66}$.

Les formes dissociées de la propriété, si elles compliquent certains mécanismes administratifs, permettent également au prince d'exercer son pouvoir d'arbitrage. Car la complexité des régimes de propriété fait naître nombre de conflits d'usage, surtout si les édifices concernés jouxtent l'espace public. Là encore, le portique, débordant sur la voie commune, apparaît comme un lieu sensible. En 1476, le duc de Milan autorise les «voisins» de la contrada de San Sisto à concéder "in emphiteosim perpetuam" un portique à Gabriele de Blanchis. A charge pour celui-ci d'y faire des travaux, "in ornamentum urbis» ${ }^{67}$. Soit que les travaux aient dépassés le cadre prévu, soit que leur avancement ait suscité de nouveaux conflits, le duc reçoit quelques temps plus tard une supplique contestant ces aménagements ${ }^{68}$. Cette protestation est signée par les voisins de Gabriele, parmi lesquels on reconnaît les plus grands noms de l'oligarchie milanaise habitant dans ce quartier de Porta Ticinese : les Brivio, Panigarola, Vicomercati, Biraghi, Piatti... Ceux-ci composent leur argumentation en deux temps : d'abord, ce portique a, d'antique mémoire, toujours servi d'agrément aux gentilshommes ${ }^{69}$. Est-il légitime, demandent les pétitionnaires, qu'un seul individu prive la collectivité d'un espace dont l'usage était commun? Car, et c'est le second point de l'argumentaire, ces aménagements privés entravent la circulation, ce qui représente une gêne pour l'en-

p. 199 sq. apporte des précisions déterminantes quant à la datation de ces statuts. Voir, comme exemple éclairant d'utilisation de cette source, L. CHIAPPA MaURI, I mulini ad acqua nel milanese (secoli X-XV), Città di Castello, 1984 (Biblioteca della "Nuova Rivista Storica», 36), p. 68 sq.

${ }^{60}$ ASCMi, Litterarum ducalium, 1462-1472, fo 184v-185r, 19 janvier 1470.

${ }^{67}$ ASMi, Registri ducali, Reg. 50, fo $265 \mathrm{v}, 20$ aout 1476. La concession se fait en deux temps : pour une partie seulement du portique le 26 mai, puis pour sa totalité le 20 aout. Les documents relatifs à cette affaire furent signalés, et partiellement publiés par P. GHINzonI, Di alcuni antichi coperti ossia portici in Milano, dans Archivio storico lombardo, IX, 1892, p. 126-140.

${ }^{68}$ ASMi, Comuni, cart. 54, s.d. Cette supplique est, comme on va le voir, nécessairement antérieure à la lettre du 3 mai 1481 concernant la même affaire.

${ }^{69}$ Ibid., "...uno coperto antiquamente et per tanto tempo che non gli hè memoria in contrario constructo per recreatione deli Zentilohomini de quella porta...» 
semble du peuple, pour les voyageurs et les étrangers, et donc une atteinte à la dignité de la ville ${ }^{70}$.

Sans doute Gabriele de Bianchis a-t-il pris connaissance de la pétition, en tout cas, il y répond point par point dans une supplique également adressée au duc ${ }^{71}$. Préalablement, il refuse à ses adversaires le droit de parler pour la communauté : eux-mêmes défendent des intérêts privés, et Gabriele glisse même qu'ils pourraient agir pour le compte d'un certain Bechino d'Abia ayant des vues sur le portique. Il rappelle, en premier lieu, que les travaux qu'il a entrepris sur le portique sont parfaitement légitimes, puisqu'il a pris soin de se munir d'une licence ducale ${ }^{72}$. Dans un second temps, il retourne à son avantage l'argument, décisif pour l'idéologie princière, de l'omamentum urbis : en réalité, ce portique était, avant qu'il lui soit concédé, un lieu sale et mal famé, ses aménagements participent donc à l'embellissement et à l'honneur de la cité73.

La réponse ducale est sans surprise. Comme en pareil cas, il nomme une commission d'experts formée du sindaco Gabriele Panigarola, d'Ambrogio Ferrario, commissaire ducal chargé des grands travaux et de cinq ingénieurs de la commune ${ }^{74}$. Ceux-ci ne peuvent évidemment revenir sur la concession ducale, qui vaut donc comme légitimation politique des travaux entrepris. On comprend mieux, au passage, combien il était finalement intéressant pour les concessionnaires de se soumettre à cette obligation de licence ducale, qui garantissait leurs droits contre toute contestation des voisins ou propriétaires. Toutefois, et "per levare ogni differentia", la commission oblige la famille de Bianchis à prendre en charge les travaux de restructuration de la voirie rendus nécessaires par les aménagements du portique.

${ }^{70}$ Ibid., "...et perchè simili cosse che fino facte ad uso di tuti non se deno destruere senza licentia de tuti, maxime considerato che questa cossa serebe non solamente in dispiacere et disconzo deli dicti supplicanti, ma ancora de tuto il populo, et tuti li viandanti et forestere li quali hano a passare per li a cavallo et a pede cum grande careze e cavalante e perchè è in loco multo streto, et Vostre Excellentie non soleno patire sia facta iniusticia ne iniurie ad alcuno, como è debita cossa».

${ }^{71}$ ASMi, Comuni, cart. 54, s.d.

${ }^{72}$ Ibid., "perché con lettere de licentia et bona dispensa delo illustrissimo duca Galeaz segnate Cichus fu legittime et solennemente investito ad livello del dicto coperto da quelli alli quali specta cioè dali vicini de Sancto Sisto".

${ }^{73} \mathrm{Ibid}$., "... quale coperto derelicto era pieno d'ogni immundicia et speluncha de assasini con grandissimi fetori in grande obprobio dela cità, la cui refectione evidenter cede non solo ornamento della cità vostra, ma etiam ad honore...»

${ }^{74}$ ASMi, Autografi, cart. 86, fasc. 39 («Giovanni Solari»), 3 mai 1481. Les quatre autres signataires sont les ingénieurs Danesio de Maineri, Petrus de Lonate, Ambrogio de Bernadiglio, Gabriele de Giringellis. 
On saisit alors les enjeux réels de la controverse : débordant sur la voie publique, le portique de Gabriele de Bianchis servait de passage couvert, de lieux de halte et de réunion. Il formait ce type d'espace intermédiaire où s'établissaient les liens entre la famille et la communauté, tel qu'il a été étudié à Rome par Henri Broise et JeanClaude Maire Vigueur ${ }^{75}$. Or, dans les dernières décennies du XVe siècle, la clôture des portiques participe à un réaménagement global de l'espace domestique, qui supprime ou condamne les espaces intermédiaires, et réorganise l'architecture intérieure autour d'une cortina et d'espaces de circulation ${ }^{76}$. Ces restructurations sont à mettre en rapport avec le nouvel usage locatif des maisons, et d'une manière plus globale, avec une transformation des modes d'habiter et des relations sociales. L'histoire de ce repli où l'espace privé est «ressaisi par le lignage», si bien décrit dans le cas vénitien par Élizabeth CrouzetPavan ${ }^{77}$, est également discernable à Milan. Les cours s'y referment sur un usage strictement privé, avec l'assentiment du pouvoir princier qui accordent ces licences d'édification ${ }^{78}$. Entre l'espace public et l'espace privé, la coupure se fait plus franche, effaçant saillies et chevauchements qui constituaient la cible privilégiée des interventions ducales. Cependant, ces mutations des espaces intermédiaires, ces glissements de frontière entre le champ public et le champ privé occasionnent à leur tour nombre de conflits d'usage que l'autorité princière est appelée à trancher, affirmant ainsi son pouvoir d'arbitrage et de contrôle ${ }^{79}$.

${ }^{75}$ H. Broise et J.-C. MAIre VIgueUr, Strutture familiari, spazio domestico $e$ architettura civile a Roma alla fine del Medioevo, dans Storia dell'arte italiana, vol. 12, Momenti di architettura, Turin, 1983, p. 97-160.

${ }^{76}$ ID., ibid. Voir également H. BRoIsE, Les maisons d'habitation à Rome aux $X V^{e}$ et $X V I^{e}$ siècles : les leçons de la documentation graphique, dans D'une ville à l'autre..., op. cit., p. 609-629.

${ }^{7}$ É. Crouzet-Pavan, op. cit., t. 1, p. 493 sq.

${ }^{78}$ Par exemple, en 1467, Raffaele de Busseto, membre du conseil secret, est autorisé à "chiudere il piazola sito prope domus habitationis», longue de 35 brassées et larges de 8 ( $20 \mathrm{~m} 60$ par $4 \mathrm{~m} 70$ environ) pourvu qu'il obtienne l'accord des voisins de sa contrada. ASMi, Litterarum ducalium, 1462-1472, Reg. 11, fo 195r, 21 janvier 1467. De même, les aménagements successifs du Palazzo Borromeo à Milan offre un exemple célèbre de ce type de restructuration architecturale. Voir L. PAtetra, L'architettura del quattrocento a Milano, Milan, 1987, p. 241-243.

${ }^{79}$ L'achèvement de ce processus historique a lieu en 1493 quand Ludovic le More ordonne la destruction de tous les portiques, balcons et saillies dans le centre de Milan. Expérience urbanistique déterminante, que nous ne pouvons étudier ici, mais sur laquelle nous cspérons revenir bientôt. Le pouvoir princier affirme avec vigueur son désir de monumentalité, en accompagnant et en facilitant une mutation foncière qui profite avant tout aux intérêts des classes dirigeantes, soucieuses de se rassembler désormais en des quartiers choisis, et de se retrancher derrière des palais dont l'emphase de la facade privée d'espaces intermédiaires exprime la coupure d'avec leurs clientèles. 
Peut-être les Sforza ont-il eu un temps le projet politique d'un contrôle global du marché immobilier dans leur capitale. De ce contrôle, on peut comprendre les motivations, appréhender les modalités, reconstituer les moyens mis en œuvre. De ce fait, les archives publiques peuvent être lues comme une source indirecte de l'histoire de la propriété. Mais les lacunes de cette documentation sont évidentes, comme le sont pareillement les défaillances de ce contrôle princier, aux mailles bien lâches. Toutefois, si les lettres de concession, licences d'édification et autres formes d'intrusion ducale dans les transactions privées sont bien loin de restituer une image fidèle de l'ensemble des structures de la propriété à Milan, elles permettent au moins de se faire une idée de la représentation qu'en avait l'autorité ducale, des enjeux qu'elle y décelait et des tendances qu'elle souhaitait appuyer ou contrarier. De ce point de vue, leur lecture peut corriger ou compléter celle que l'on fait habituellement des statuts communaux et des textes normatifs. Certes, le pouvoir cherche à délimiter, à borner, à séparer : ainsi, l'espace public sera défendu contre les empiétements du privé, le droit du concessionnaire protégé contre les abus du propriétaire, les régimes de propriété distingués et réglementés. Mais en même temps, la marge, le chevauchement, l'imbrication constituent également des lieux où s'affirme et s'exerce le pouvoir du prince. C'est à partir des saillies et des débordements de l'architecture domestique sur la voie publique que le prince tente d'imposer son contrôle et, à son tour, de «mettre un pied" dans l'édification privée. C'est en s'insérant dans les méandres de la propriété partielle qu'il obtient un droit de regard sur les transactions immobilières. C'est, enfin, en tirant partie des conflits que font naître les transformations des usages sociaux de l'espace urbain qu'il peut user de son pouvoir d'arbitrage et défendre sa conception de l'ornamentum urbis, qui veut dire à la fois embellissement de la ville du prince et aisance de ceux qui le servent. 